\title{
SAMMLUNG METZLER
}

M 1 Raabe Einfübrung in die Bücherkunde

M 3 Meisen Altdeutsche Grammatik II: Formenlebre

M 4 Grimm Bertolt Brecht

M 5 Moser Annalen der deutschen Sprache

M 6 Schlawe Literarische Zeitschriften 1885-1910

M 7 Weber/Hoffmann Nibelungenlied

M 8 Meyer Eduard Mörike

M 9 Rosenfeld Legende

M 10 Singer Der galante Roman

M 12 Nagel Meistersang

M 13 Bangen Die schriftliche Form germanist. Arbeiten

M 14 Eis Mittelalterliche Fachliteratur

M 15 Weber/Hoffmann Gottfried von Straßburg

M 16 Lüthi Märchen

M 17 Wapnewski Hartmann von Aue

M 18 Meetz Friedrich Hebbel

M 19 Schröder Spielmannsepik

M 20 Ryan Friedrich Hölderlin

M 22 Danzel Zur Literatur und Philosophie der Goethezeit

M 24 Schlawe Literarische Zeitschriften 1910-1933

M 25 Anger Literarisches Rokoko

M 26 Wodtke Gottfried Benn

M 27 von Wiese Novelle

M 28 Frenzel Stoff-, Motiv- und Symbolforschung

M 29 Rotermund Christian Hofmann von Hofmannswaldau

M 30 Galley Heinrich Heine

M 31 Müller Franz Grillparzer

M 32 Wisniewski Kudrun

M 33 Soeteman Deutsche geistliche Dichtung des 11. u. 12. Jh.s

M 34 Taylor Melodien des Mittelalters I: Darstellung

M 35 Taylor Melodien des Mittelalters II: Materialien

M 36 Bumke Wolfram von Eschenbach

M 37 Engel Handlung, Gespräch u. Erzäblung. Faksimiledruck

M 38 Brogsitter Artusepik

M 39 Blanckenburg Versuch über den Roman. Faksimiledruck

M 40 Halbach Walther von der Vogelweide

M 41 Hermand Literaturwissenschaft und Kunstwissenschaft

M 43 Glinz Deutsche Syntax 


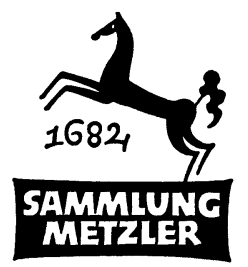

REALIEN ZUR LITERATUR ABT. E: POETIK 
ELISABETH FRENZEL

\section{Stoff-, Motiv- und Symbolforschung \\ 4., durchgesebene und ergänzte Auflage}


1. Aufl. ( 1.- 5. Tsd.) 1963

2. Aufl. ( 6.-10. Tsd.) 1966

3. Aufl. (11.-15. Tsd.) 1970

4. Aufl. (16.-20. Tsd.) 1978

CIP-Kurztitelaufnahme der Deutschen Bibliothek

Frenzel, Elisabeth:

Stoff-, Motiv- und Symbolforschung. -

4., durchges. u. erg. Aufl. - Stuttgart:

Metzler, 1978.

(Sammlung Metzler; M 28: Abt. E, Poetik)

ISBN 9.78-3-476-14028-9

ISBN 978-3-476-99315-1

ISBN 978-3-476-99314-4 (eBook)

DOI 10.1007/978-3-476-99314-4

M 28

(C) 1978 Springer-Verlag GmbH Deutschland

Ursprünglich erschienen bei J. B. Metzlersche Verlagsbuchhandlung und Carl Ernst Poeschel Verlag GmbH in Stuttgart 1978 
Herkunft und Geschichte der Begriffe Stoff, Motiv, SYMBOL ................. 1

ENTWICKLUNG DES FoRSCHUNGSGEBIETES ........ . . 3

Stoff- und Motivgeschichte . . . . . . . . . . . . . . 3

Literarkritische Ansätze - Volksliteraturforschung . . . . . 3

Die Scherer-Schule - Diltheys Motivenlehre und die vergleichende Literaturwissenschaft . . . . . . . . 5

Abwertung der Stoff- und Motivgeschichte durch die geistesgeschichtliche Methode . . . . . . . . . 7

Neuorientierung der Stoff- und Motivgeschichte in der modernen Poetik ............. . 9

Symbolforschung . . . . . . . . . . . . . . . . . . 14

Christliche Symbolkunde - Asthetische Begriffsauslotung Romantische Mythusforschung - Philosophische Ausweitung des Begriffs Symbol ... . . . . . . . . . . Die Literatur des Symbolismus - Symboldeutung der Psychoanalyse . . . . . . . . . . . . . . . . . . 17 Literaturwissenschaftliche Symbolforschung . . . . . 21

Systematische Klärung der Begriffe und ihre Abgrenzung GEGEN VER WANDTE UND BENACHBARTE Begriffe . . . . . . 24

Stoff . . . . . . . . . . . . . . . . 24

Motiv . . . . . . . . . . . . . . . . . . 29

Symbol ................. 35

MÖGLIChKeITEN UND BISHeRIGe ERgebnisse DER STOFF-, Motivund SYMBOLFORSCHUNG . . . . . . . . . . . . . 46

Das Verbältnis des Dichters zu Stoff, Motiv, Symbol . . . . 47

Topik - Motivkonstanz - Routinemäßige Motivübernahme und Absinken von Motiven . . . . . . . . . . . . . . . 49

Stoffübernahme und Stoffveränderung . . . . . . . . 53

Prävalenz bestimmter Motive und Symbole bei einzelnen Autoren . . . . . . . . . . . . 58

Beziebungen zwischen Nationalliteratur und Stoff, Motiv, Symbol. . . . . . . . . . . . . . 65

Geistes- und sozialgeschichtliche Perspektiven des Forschungszweiges . . . . . . . . . . . . . . . . . 68 Die Einheit der literarischen Generationen und Epochen . . 68 Stoff wandlungen und Motivmutation . . . . . . . . 71 Traditionelle, erhellende und hermetische Symbole . . . . . 76 
Ergiebigkeit des Forschungszweiges für Zuweisungs-, Herkunftsund Datierungsfragen . . . . . . . . . . . 81

Leistungen der Stoff-, Motiv- und Symbolforschung für die Poetik . . . . . . . . . . . . . . . . 82

Gattungsaffinität und innere Struktur von Stoffen und Motiven . . . . . . . . . . . . . . . 82 Strukturveränderung bei Gattungswechsel . . . . . . . 91 Wachstum und Wahlverwandtschaft von Stoffen und Motiven ................ . 93 Struktur und Funktion von Symbolen . . . . . . . . . . 96 Symboldeutung . . . . . . . . . . . . . 100

Autoren- Und TITELREgISTER . . . . . . . . . . . . 107

SACHREgISTER . . . . . . . . . . . . . . 111 


\section{AbKüRZUngen}

AfnSpr. Archiv für das Studium der neueren Sprachen und Lite-

DLZ Deutsche Literaturzeitung

DtPhil.i.A. Deutsche Philologie im Aufriß

DVjs. Deutsche Vierteljahrsschrift für Literaturwissenschaft

Euph. Euphorion

GRM Germanisch-Romanische Monatsschrift

RL Reallexikon der deutschen Literaturgeschichte

WA Goethes Werke, Weimarer Ausgabe

ZfdA Zeitschrift für deutsches Altertum

ZfDk. Zeitschrift für Deutschkunde

ZfdPh. Zeitschrift für deutsche Philologie

ZfdU Zeitschrift für den deutschen Unterricht 